\title{
Implementation of an Online Software Tool for the Analytic Hierarchy Process (AHP-OS)
}

\begin{abstract}
The analytic hierarchy process (AHP) remains a popular multi-criteria decision method. The author has implemented a free, web-based AHP online system with noteworthy features, allowing for the detailed analysis of decision problems. Beside standard functions like flexible decision hierarchies, support to improve inconsistent judgments, alternative evaluation and sensitivity analysis, the software can handle group inputs, calculate group consensus based on Shannon $\alpha$ and $\beta$-entropy, and estimate weight uncertainties based on randomized small variations of input judgments. In addition, different AHP judgment scales can be applied a posteriori, and alternative evaluation can be done using the weighted sum (WSM) or weighted product model (WPM). This flexibility opens up opportunities to study the classical AHP and decision projects under various parameters. The author's intention was to provide a complete and free software tool for educational and research purposes, where calculations and algorithms are well documented and all input data and results can be exported in an open format for further processing or presentation. The article describes the basic structure of the software and highlights key features of its implementation. The full description of all underlying methods and algorithms is available from the author's website.
\end{abstract}

Keywords: Multi-criteria decision making, Analytic hierarchy process, AHP Software, AHP online system, AHP Group decision making.

\section{Introduction}

The analytic hierarchy process (AHP) was developed by T. L. Saaty in the 1980 and remains a widespread multi-criteria decision method (MCDM). Based on pairwise comparison inputs, weights are calculated by finding the dominant right eigenvector (EV) of a positive reciprocal decision matrix. Calculations for simple decision problems can be done with a spreadsheet program (Goepel, 2013), but for more complex decision problems a software tool is needed.

\section{Literature Review}

There are several AHP software packages available on the market, and some of them have been described or were compared in the literature (Ishizaka, Labib, 2009, Ossadnik, Kaspar, 2013, Siraj et al., 2015). Most of these software packages are realized as commercial products and are focused on applications. Often the underlying methods and algorithms are not fully transparent to users.

\section{Objectives}

The author's intention was to provide a complete and free AHP software tool for educational and research purposes, where methods and algorithms are well documented 
and validated. The software should provide a variety of options and features to analyze and study AHP projects under different parameters. As a result, a web-based AHP online system (AHP-OS) was developed and is available in its full functionality to any user for non-commercial purposes.

\section{Methodology}

Implementation of the software was done in PHP, an open source general-purpose, object oriented scripting language that is especially suited to web development. Database functions are implemented using Structured Query Language (SQL), the most widely used database language with available open source database engines like SQLite or MariaDB. The whole package was developed from scratch, using only a few other available open source packages for general supporting functions, like sending of e-mails or user login and registration. Functions and features of AHP-OS include

- Flexible definition of decision hierarchies as text input, following a simple syntax with multi-language support using Unicode character coding.

- Weight calculation (hierarchy mode) and alternative evaluation (alternative mode) using the AHP eigenvector method.

- Pairwise comparison input, highlighting the top-3 most inconsistent judgments.

- A posteriori application of different AHP judgment scales.

- Group decision making using weighted geometric mean aggregation of individual judgments (WGM-AIJ).

- Group consensus calculation based on Shannon $\alpha$ and $\beta$-entropy.

- Weight uncertainty estimation using Monte Carlo simulation.

- Sensitivity analysis.

- Weighted sum model (WSM) and weighted product model (WPM) for the aggregation of alternatives.

- Export of input and result data as comma separated value (CSV) files for further processing or presentation in a spreadsheet program.

\section{Implementation}

The software has a modular structure and is implemented using object oriented programming (OOP). It consists of five major objects classes:

1. ahp class for all basic AHP calculations (pairwise comparisons, eigenvector),

2. ahpHierarchy class for decision hierarchy related functions,

3. ahpDb class for database handling,

4. ahpGroup class for result calculations and

5. ahpAdmin class for all administrative functions, like user and project administration.

These classes contain all AHP methods, and in the following implementation of some major functions is highlighted.

\subsection{Decision Hierarchies}

The definition of decision hierarchies is implemented as plain text input, consisting of a sequence of statements following a simple syntax, resulting in the following advantages: 
- Hierarchy definitions in plain text form can be modified or duplicated using copy and paste functions, and they can be easily archived as text files.

- Unicode character coding allows for multi-language support; languages like Chinese, Korean, Thai, etc. are supported.

- Weights can be partly or completely predefined within a hierarchy text. This is useful when weights are already known, e.g. from a previous project, or when weights are derived from other MCDM methods.

The syntax is defined as follows:

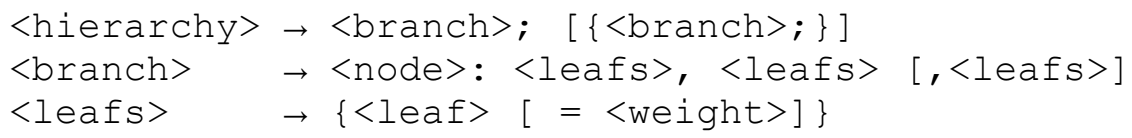

Fig. 1 shows a simple example of a two level decision hierarchy in AHP-OS. In the first level (main criteria), weights for the two criteria are predefined as $30 \%$ and $70 \%$. For the sub-criteria weights are automatically set to a default value $1 / n_{\text {leaf }}$.

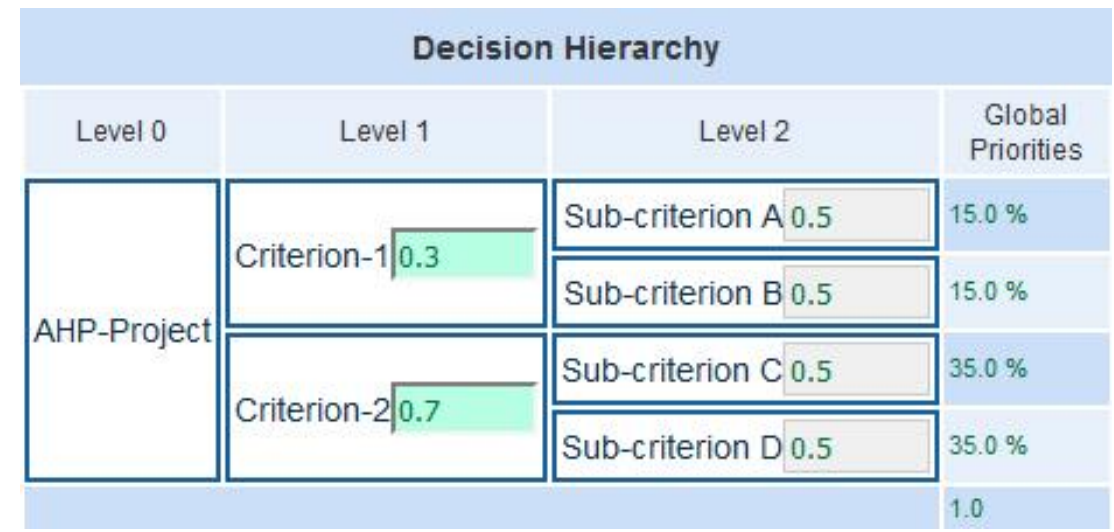

Fig. 1 Example of a decision hierarchy in AHP-OS. This hierarchy has 2 levels, 3 nodes (AHP priority vectors) and 4 leafs.

This hierarchy in fig. 1 is defined by the following text input:

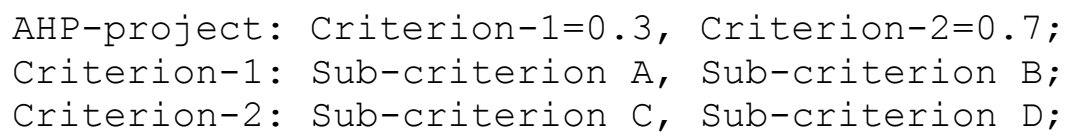

All methods related to the decision hierarchy are coded in the ahpHierarchy class of the software. The class includes a parser to translate the hierarchy text into a multidimensional array. The parser checks for syntax errors and cleans the input text from redundant and impermissible characters. Supporting functions to extract nodes, branches or leafs from the hierarchy are included in this class.

\subsection{Database and Data Structure}

The software uses the PHP data object (PDO) interface to SQL databases. The current implementation can handle two SQL servers, either open source MariaDB, or the public 
domain SQLite database engine. The implemented database structure comprises of four database tables:

1. Users: table for user registration and login. Registered users can define, store and manage their own AHP projects.

2. Projects: table of AHP projects with a session code, project name, project description and hierarchy definition. Its foreign key is the user name from the table Users.

3. Judgments $(p w c)$ : table containing all pairwise comparisons with the decision makers' name and nodes of the decision hierarchy. Foreign key is the session code from the table Projects.

4. Alternatives: table with alternative names. Foreign key is the session code from the table Projects.

The database structure minimizes redundancies and keeps the database slim. In order to identify projects, each project gets a unique six character session code. For group decision making participants' judgments are stored in the $p w c$ table. All pairwise comparisons are stored in a simple integer format. Participants can start to input their judgments by opening a link provided by the project author (registered user). The $a h p D b$ software class handles the interfacing with the SQL database server. Results are not stored in the database, they are calculated on-the-fly using methods in the software class ahpGroup.

\subsection{AHP Judgment Scales}

Over the last decades a variety of judgment scales, different from Saaty's fundamental one to nine scale, have been proposed. In AHP-OS pairwise comparisons are stored with their original judgment values, users therefore can apply different scales a posteriori and study the effect of different scales on the resulting weights. Ten different scales are implemented. A comparison of these scales, based on weight boundaries and weight ratio, weight uncertainty and weight dispersion, is given by Goepel (2017).

\subsection{Group Decision Making and Group Consensus}

The weight distribution of criteria among different decision makers is analyzed using Shannon entropy and its partitioning in two independent components ( $\alpha$ and $\beta$-entropy) to derive an AHP consensus indicator (Goepel, 2013). The consensus indicator ranges from $0 \%$ (no consensus) to $100 \%$ (full consensus). The whole range is categorized into the five categories very low, low, moderate, high and very high.

\subsection{Weight Uncertainties}

As shown by Goepel (2017) weight uncertainties due to rounding of judgment values can exceed 10\%, and could impact the results of a decision. In order to get an estimation of uncertainties, randomized variations of the original judgments are generated (Monte-Carlo simulation). The program provides estimated weight uncertainties for all criteria or alternatives.

\section{Limitations and Future Extensions}

AHP-OS is implemented to handle the classical AHP, e.g. it is not designed to handle fuzzy inputs or other variations of the original AHP. A few extensions are under consideration. 
As a major extension a cluster analysis of group consensus is planned. The concept of Shannon entropy and its partitioning in $\alpha$ and $\beta$-entropy allows to identify clusters or subgroups of decision makers with high consensus within the whole group of decision makers. This could help to gain a deeper insight into the group decision making processes.

\section{Conclusions}

The AHP-OS software was originally released in 2014. Features described in this paper were developed gradually. With the currently implemented functionality the software tool has reached a state, where it covers most of the possible options for the classical analytic hierarchy process. It allows for a wide range of parameter variations to analyze and study a specific project under different aspects. This and the possibility of transparent data export makes it an ideal tool for study, education and further research. Up to the date of writing this paper more than 5500 users have registered for the software, and on average there are 750 active users over a three months period. AHP projects handled with AHP-OS cover a wide range of applications like healthcare, climate, risk assessment, supplier selection, hiring, IT, marketing, environment, transport, project management, manufacturing or quality assurance. Numerous projects are used to acquire group inputs, the number of participants goes up to 320 in a single projects. Multi-language support is utilized by users for languages like Chinese, Korean, Russian, Hebrew, Greece, Thai, Vietnamese or Arabic. Lecturers at universities make use of AHP-OS as a tool for their students, when teaching multi criteria decision making methods.

Overall the number of software bugs could be kept reasonable low, more often users' feedback and questions relate to the understanding of the AHP and can be solved straightforwardly. The main challenges in maintaining the software are updates of the underlying open source tools due to security reasons, or smaller updates requested by users. Because of the complexity of the software changes require extensive testing and validation.

\section{Key References}

Goepel, K. D. (2013). Implementing the Analytic Hierarchy Process As A Standard Method For Multi-Criteria Decision Making In Corporate Enterprises - A New AHP Excel Template with Multiple Inputs. Proceedings of the International Symposium on the Analytic Hierarchy Process, Kuala Lumpur.

Goepel, K. D. (2017). Comparison of Judgment Scales of the Analytical Hierarchy Process - A New Approach, submitted for consideration in International Journal of Information Technology and Decision Making.

Ishizaka,A., Labib, A. (2009) Analytic Hierarchy Process and Expert Choice: Benefits and Limitations, OR Insight, 22(4), p. 201-220.

Ossadnik, W., Kaspar, R. (2013). Evaluation of AHP software from a management accounting perspective. Journal of Modelling in Management, Vol. 8 No. 3, pp. 305-319.

Siraj, S., Mikhailov, L., Keane, J. A., PriEsT (2015). An Interactive Decision Support Tool To Estimate Priorities From Pairwise Comparison Judgments, International Transactions in Operational Research, Vol. 22, Issue 2, 217-235. 